\title{
Vom Mitsein der Toten - Gedenksitzung der AG „Sprache und Ethik“ für und mit Theda Rehbock (1957-2021)
}

\section{Online-Veranstaltung am 27.04.2021}

\author{
Elsa Romfeld • Alice Schwab
}

Angenommen: 14. Juli 2021 / Online publiziert: 22. Juli 2021

(C) Der/die Autor(en) 2021

Am 27. April 2021 fand die Online-Gedenksitzung der AG „,Sprache und Ethik“ für unsere liebe Freundin und geschätzte Kollegin Frau Prof. Dr. Theda Rehbock statt, die zu Beginn dieses Jahres nach längerer Krankheit verstorben ist. Sie hat unsere AG im Jahr 2007 gegründet, über neun Jahre geleitet und mit ihrer sehr persönlichen Art geprägt. Es war ihr ein Herzensanliegen, für die sprachlichen Aspekte im medizinethischen Kontext zu sensibilisieren; dafür hat sie Menschen aus unterschiedlichsten Bereichen gewonnen und begeistert. Auch nach Weitergabe der Koordination 2017 blieb sie Mitglied der AG und nahm an deren Arbeit und Entwicklung Anteil bis zu ihrem Tod.

Als Zeichen der Würdigung und der über den Tod hinaus bestehenden Verbindung mit Theda hatten wir zur Auseinandersetzung mit ihrem Text „Der Tod und die Toten - philosophisch betrachtet“ (Rehbock 2018) eingeladen. Langjährige Wegbegleiter und Freundinnen gaben die im Folgenden kurz zusammengefassten persönlichen und fachlichen Impulse, die jeweils im Anschluss lebhaft diskutiert wurden:

- Prof. Dr. Hans Werner Ingensiep (Duisburg-Essen):

Theda Rehbock habe ich im Jahr 2008 während der Vorbereitung eines BMBF-

Projektes zum Thema „Sprache und Sinn in Grenzsituationen des Lebens“ am Institut für Medizin- und Wissenschaftsgeschichte an der Universität zu Lübeck kennen und schätzen gelernt. Auf der Suche nach philosophischer Unterstüt-

\footnotetext{
Elsa Romfeld, M.A. ( $₫)$

Fachgebiet Geschichte, Theorie und Ethik der Medizin, Medizinische Fakultät Mannheim, Universität Heidelberg, Ludolf-Krehl-Straße 13-17, 68167 Mannheim, Deutschland

E-Mail: elsa.romfeld@medma.uni-heidelberg.de

Dr. med. Alice Schwab, MAE

St. Franziskus-Hospital, Münster, Deutschland
} 
zung wurde ich durch einen Hinweis von Heike Baranzke auf die Schriften von Theda Rehbock aufmerksam. Aus diesem Projekt ging das mit ihr zusammen herausgegebene Buch „Die rechten Worte finden ... “ (Ingensiep und Rehbock 2009) hervor. Es wurde zugleich zur Keimzelle für den bis heute existierenden interdisziplinären Arbeitskreis „,Sprache und Ethik“.

Mein konkreter Zugang waren aktuelle Diskussionen und Internetkommentare zum Fall der amerikanischen Wachkomapatientin Terri Schiavo im Jahr 2005: „Falls ich irgendwann mal als hirntoter Fleischklops dahinvegetieren sollte, würde ich es aus heutiger Sicht begrüßen, wenn man mich nicht krampfhaft am ,Leben " erhalten würde“ (Ingensiep 2009, S. 131), aber auch Reflexionen von Kant, Nietzsche, Jonas oder Singer zu ähnlichen Grenzsituationen des Lebens. Theda Rehbock war bei der Analyse eine konstruktive und unermüdliche Disputantin. Sie trieb das Projekt ebenso wie später den Arbeitskreis engagiert voran: „Es ist eine Demütigung, wenn einem Patienten abgesprochen wird, dass er ein Mensch ist" (Mertz 2008). Mit diesem Satz fasste sie damals für die Lübecker Tageszeitung ihr tiefes ethisches Anliegen zusammen, das ihren eigenständigen Lebensweg bis zuletzt bestimmte.

- Markus Greß-Heister, M. A., LL.M. und Dr. Elisabeth Heister (Kaiserlautern):

Für die Philosophin Theda Rehbock war es immer ein zentraler Argumentationspunkt, die Sprache in der Philosophie als mehr als ,ein bloß äußeres Element der Mitteilung “ (Rehbock 2009, S. 9) zu betrachten. Im Sinne Wittgensteins, dass alle Philosophie Sprachkritik darstellt (Wittgenstein 1960, S. 33), ist der Ausgangspunkt philosophischen Denkens Sprache als Lebenselement des Denkens, der menschlichen Erkenntnis und Selbsterkenntnis, aber auch als Sprache selbst Instrument menschlichen Lebens und Handelns (vgl. Rehbock 2009, S. 9). Es bestand für Theda Rehbock immer ein deutlicher und enger Zusammenhang zwischen medizinischer Sprache und Alltagssprache, der sich auch in der Bedeutung literarischer Sprachmittel in der Sprache der Ethik niederschlägt. Mit Merleau-Ponty kritisiert sie in ihrem Werk immer wieder die Wissenschaftssprache in ihrer objektivistischen Auffassung und Reduktion auf „langue“. Sie setzt dagegen die Sprache als „parole“, als lebendige Rede, Gegenrede und Sprechen.

Auch die immanente leiblich biologische Verankerung der Sprache als „matter-involving " war für Theda Rehbock klar in die theoretischen Überlegungen zu Ethik in der Medizin einzubeziehen. Für sie bedeutet Sprache und die Versprachlichung philosophischer Gedanken das Erzeugen brauchbarer intersubjektiver Vorstellungen durch Sprache als Lernprodukt aus sozialer Interaktion: Wörter bedeuten Begriffe, Begriffe deuten auf Vorstellungen und Konzepte. Wortbedeutungen sind subjektive Konstrukte, die eben nur relative Werturteile zulassen. Theda Rehbock geht in ihren sprachphilosophischen Aussagen immer von Sprachlichkeit als Grunddimension personalen Sinnhorizonts im Verhältnis zum Anderen als personalem Gegenüber in einem gemeinsamen Weltbezug aus. Normengebend war für sie hier das Verstehen in Beziehung zur Beobachtung der Beziehung zueinander. Hier kann Theda Rehbock durch ihre Haltung als 
Übersetzerin oder Vermittlerin in Richtung auf eine konstruktivistische Haltung gesehen werden, ohne dass sie diese jemals ausdrücklich eingenommen hat.

- Dr. Mechthild Herberhold (Altena):

Tote sind weiterhin Personen, so die Position von Theda Rehbock - und dabei argumentiert sie explizit immanent: „Auch der [...] Tote bleibt die Mutter, der Freund oder auch der Fremde, in dessen Lage wir jederzeit selbst geraten könnten und dem gegenüber unser Verhalten nicht moralisch gleichgültig ist" (Rehbock 2002, S. 142). Bilder von Theda Rehbock aus verschiedenen Jahren und Portraits von Menschen vor und nach dem Tod (vgl. Lakotta und Schels 2004) verdeutlichen, dass Menschen erkennbar bleiben. Die Toten sind weiterhin im Mitsein präsent und die Beziehung der Lebenden zu den Verstorbenen ist mehr als die Erinnerung an frühere Ereignisse: „Das Leben der Lebenden mit dem Toten als Person - nicht nur mit seinem Körper! - geht in anderer Form weiter" (Rehbock 2018, S. 35). Ein Beispiel: Die Auseinandersetzung mit dem Text, die Erstellung eines Impulses, das Gedenktreffen der AG Sprache - all das entwickelt meine Beziehung zu Theda Rehbock auch nach ihrem Tod weiter. Werte gegenüber Verstorbenen gilt es aktiv zu füllen. Fürsorge kann etwa im Waschen, Ankleiden und Bestatten gelebt werden. Um Respekt geht es in Nachrufen, beim Sprechen über Verstorbene oder im Umgang mit ihren Werken. Vertraulichkeit drückt sich im Umgang mit Tagebüchern und Briefen aus. Lebende lassen Toten Gerechtigkeit widerfahren durch Verurteilung der Täter, Rehabilitation oder Entschädigung. Sicher sind alle Handlungen auch für die Trauernden wichtig im Sinne einer Gestaltung der eigenen Traueraufgaben (vgl. Worden 2011), aber - das Personsein von Toten vorausgesetzt - eben nicht nur. Nicht zuletzt sagt die Art und Weise, wie wir mit den Toten umgehen, etwas über uns selbst aus, und es beeinflusst bzw. verändert uns in die eine oder andere Richtung.

- Prof. Dr. Margit Sutrop (Tartu, Estland):

Unsere Wege kreuzten sich 1992 in Konstanz, wo Theda gerade Philosophie unterrichtete und ich promovierte. Ich habe ihr Seminar zur Phänomenologie besucht und damit hat unser philosophischer Austausch angefangen, der 29 Jahre lang gedauert hat. Sie hat mich mehrmals in Tartu besucht und in meinem Ethikzentrum Vorträge und Seminare gehalten. Sie war von Estland so begeistert, dass sie im Herbst 2019 hier eine neue Heimat gefunden hat. Theda hat davon geträumt, dass ihr Haus in Susimetsa zu einem offenen Zentrum für Philosophie wird, wo die Menschen die Möglichkeit haben, Bücher zu schreiben und zugleich im Garten zu arbeiten. Leider blieb die Gründung eines philosophischen Zentrums in Susimetsa unvollendet.

Theda war eine hervorragende Kennerin der Kant'schen Philosophie, aber sie hat sich auch gut in der phänomenologischen Tradition sowie in der antiken Philosophie ausgekannt. In ihrem Artikel „Der Tod und die Toten“ argumentiert Theda Rehbock, dass auch Toten der Status einer Person zukomme. Die 
Voraussetzung dafür ist die Auffassung des Todes nicht als Ende des biologischen oder mentalen Lebens, sondern als Grenzsituation. Da der Verstorbene den Status einer Person habe, seien die Hinterbliebenen verpflichtet, den Verstorbenen in seiner Menschenwürde als Rechtssubjekt zu achten. Die Menschenwürde basiert auf Autonomie. Die Verpflichtung, die Autonomie und den Willen anderer zu respektieren, gilt für die Lebenden ebenso wie für die Toten. Den anderen, darunter den Toten, zu respektieren, bedeutet auch, den anderen in seiner Andersartigkeit und Fremdheit zu respektieren.

Wie können wir wissen, was der wahre Wille der anderen Person ist? Theda Rehbock weist darauf hin, dass der Wille nicht immer mit dem rationalen Willen identifizierbar ist, noch ist er sprachlich kommunizierbar. Unter bestimmten Umständen könne jedoch die tatsächliche Willensäußerung einer Person in Konflikt mit einem zuvor geäußerten Willen stehen und es daher notwendig sein, in das Verhalten der anderen Person einzugreifen, wenn diese selbst nicht in der Lage ist, die Folgen und Risiken ihres Verhaltens zu verstehen. Doch paternalistische Handlungen können nicht allein auf Grundlage der Prinzipien von Wohlwollen (benevolence) und Nicht-Schaden (non-maleficence) gerechtfertigt werden. Die Autonomie bleibt für sie aber immer noch das Leitmotiv. Theda Rehbock dachte viel über den Tod nach - philosophisch, aber wenig praktisch. Das Ende des Lebens - ein so schnelles Ende - kam für sie unerwartet. Jetzt müssen ihre Freunde und ihre Familie darüber nachdenken, was ihr Wille war und wie er umgesetzt werden sollte. Wenn wir aus irgendeinem Grund von der exakten Ausführung ihres Willens abweichen, fragen wir uns, ob sie unserem paternalistischen Eingriff zugestimmt haben könnte. Denn wir wissen, dass dies das Wichtigste für Theda war.

Wir danken allen, die zum Gelingen dieser Veranstaltung beigetragen haben. Die vielen positiven Rückmeldungen haben uns gezeigt, dass die Gedenksitzung wichtig war, um Raum zu geben für Erinnerungen, den Austausch über Erlebnisse der Vergangenheit, sowie den Blick in die Zukunft zu ermöglichen. Für unsere AG „Sprache und Ethik“ bedeutet dies den Auftrag, weiterhin den besonderen Fokus auf die sprachlichen Herausforderungen im medizinethischen Diskurs zu legen. Wir danken Theda für ihr Sein, ihren großen Einsatz und die vielen Anregungen, die unsere Arbeit auch künftig prägen werden. Mögen uns ihre guten Gedanken und ihr wacher Blick stets begleiten.

Funding Open Access funding enabled and organized by Projekt DEAL.

Open Access Dieser Artikel wird unter der Creative Commons Namensnennung 4.0 International Lizenz veröffentlicht, welche die Nutzung, Vervielfältigung, Bearbeitung, Verbreitung und Wiedergabe in jeglichem Medium und Format erlaubt, sofern Sie den/die ursprünglichen Autor(en) und die Quelle ordnungsgemäß nennen, einen Link zur Creative Commons Lizenz beifügen und angeben, ob Änderungen vorgenommen wurden.

Die in diesem Artikel enthaltenen Bilder und sonstiges Drittmaterial unterliegen ebenfalls der genannten Creative Commons Lizenz, sofern sich aus der Abbildungslegende nichts anderes ergibt. Sofern das betreffende Material nicht unter der genannten Creative Commons Lizenz steht und die betreffende Handlung nicht nach gesetzlichen Vorschriften erlaubt ist, ist für die oben aufgeführten Weiterverwendungen des Materials die Einwilligung des jeweiligen Rechteinhabers einzuholen. 
Weitere Details zur Lizenz entnehmen Sie bitte der Lizenzinformation auf http://creativecommons.org/ licenses/by/4.0/deed.de.

\section{Einhaltung ethischer Richtlinien}

Interessenkonflikt E. Romfeld und A. Schwab geben an, dass kein Interessenkonflikt besteht.

Ethische Standards Für diesen Beitrag wurden von den Autoren keine Studien an Menschen oder Tieren durchgeführt.

\section{Literatur}

Ingensiep HW (2009) „Vegetieren?“ Anthropologische und bioethische Dimensionen der Sprache in Grenzsituationen des Lebens. In: Ingensiep HW, Rehbock T (Hrsg) „Die rechten Worte finden...“. Sprache und Sinn in Grenzsituationen des Lebens. Königshausen \& Neumann, Würzburg, S 123-155

Ingensiep HW, Rehbock T (Hrsg) (2009) „Die rechten Worte finden...“. Sprache und Sinn in Grenzsituationen des Lebens. Königshausen \& Neumann, Würzburg

Lakotta B, Schels W (2004) Noch mal leben vor dem Tod. Wenn Menschen sterben. Deutsche VerlagsAnstalt, München

Mertz I (2008) Wissenschaftler diskutieren in Lübeck über die Verantwortung von Sprache. Lübecker Nachrichten vom 8. August 2008: S 16

Rehbock T (2002) Autonomie - Fürsorge - Paternalismus. Ethik Med 14:131-150

Rehbock T (2009) Person und Sprache. Zur Bedeutung philosophischer Sprachkritik für die Ethik. In: Ingensiep HW, Rehbock T (Hrsg) „,Die rechten Worte finden ...“. Sprache und Sinn in Grenzsituationen des Lebens. Königshausen \& Neumann, Würzburg, S 39-58

Rehbock T (2018) Der Tod und die Toten - philosophisch betrachtet. In: Rehmann-Sutter Ch (Hrsg) Was uns der Tod bedeutet. Kadmos, Berlin, S 31-43

Wittgenstein L (1960) Tractatus logico-philosophicus. Suhrkamp, Frankfurt

Worden WJ (2011) Beratung und Therapie in Trauerfällen. Ein Handbuch. Huber, Bern 